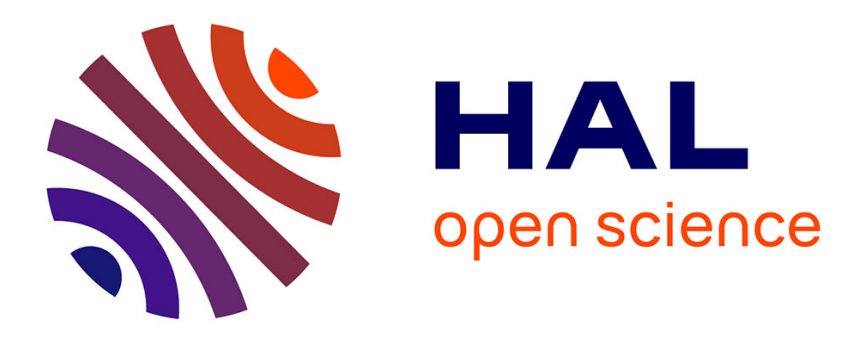

\title{
Multiple Point of Interest Discovery and Coverage with Mobile Wireless Sensors
}

\author{
Milan Erdelj, Valeria Loscrì, Enrico Natalizio, Tahiry Razafindralambo
}

\section{To cite this version:}

Milan Erdelj, Valeria Loscrì, Enrico Natalizio, Tahiry Razafindralambo. Multiple Point of Interest Discovery and Coverage with Mobile Wireless Sensors. Ad Hoc Networks, 2013, 11 (8), pp.2288-2300. 10.1016/j.adhoc.2013.04.017 . hal-00834147

\section{HAL Id: hal-00834147 https://hal.inria.fr/hal-00834147}

Submitted on 14 Jun 2013

HAL is a multi-disciplinary open access archive for the deposit and dissemination of scientific research documents, whether they are published or not. The documents may come from teaching and research institutions in France or abroad, or from public or private research centers.
L'archive ouverte pluridisciplinaire HAL, est destinée au dépôt et à la diffusion de documents scientifiques de niveau recherche, publiés ou non, émanant des établissements d'enseignement et de recherche français ou étrangers, des laboratoires publics ou privés. 


\title{
Multiple Point of Interest Discovery and Coverage with Mobile Wireless Sensors
}

\author{
Milan Erdelj $^{\mathrm{a}}$, Valeria Loscrí ${ }^{\mathrm{b}}$, Enrico Natalizio $^{\mathrm{c}}$, Tahiry Razafindralambo $^{\mathrm{a}}$ \\ ${ }^{a}$ Inria Lille - Nord Europe, France \\ milan.erdelj@inria.fr, tahiry.razafindralambo@inria.fr \\ ${ }^{b}$ DEIS University of Calabria, Italy \\ vloscri@deis.unical.it \\ ${ }^{c}$ Lab. Heudiasyc-UMR CNRS 7253, Université de Technologie de Compiègne, France \\ enrico.natalizio@hds.utc.fr
}

\begin{abstract}
Environmental monitoring has become a typical application of wireless sensor networks. The concept of monitoring certain Points of Interest (PoIs) instead of the whole sensor field helps in reducing the costs of the deployment and improving the performance in terms of coverage. However, the problems of multiple PoI discovery, coverage and data report are still solved separately and there are no works that combine the aforementioned problems into a single deployment scheme. In this work, we present a novel approach for mobile sensor deployment, where we combine multiple PoI discovery and coverage with network connectivity preservation in order to capture the dynamics of the monitored area. Furthermore, we derive analytical expressions for circular movement parameters and examine the performance of our approach through extensive simulation campaigns.
\end{abstract}

Keywords: reactive networks, mobile sensors, deployment

\section{Introduction}

Wireless Sensor Networks (WSN) have become a part of modern everyday life since they can provide information such as temperature, humidity, presence and movement detection through one or many sensing modules (static or mobile wireless sensors) deployed over the area of interest. WSN have received a lot of attention over the last decade above all in improving the deployment quality [14, 25], self-organization $[11,31]$, energy efficiency [19], communication aspects [34, 35], and the overall reliability and security [7]. A typical application of WSN is environmental monitoring. The sensors have to be deployed and placed on strategic locations to monitor the area of interest. In many cases, monitoring the whole area might be unnecessary. Therefore, monitoring some points of interest increases the sensing performance and reduces the deployment cost. Controlled mobility of sensors adds a new design primitive that needs to be carefully exploited [33]. When sensors have motion capabilities, monitoring only some PoIs instead of the whole area also permits time dependent coverage. 
Very often environmental monitoring applications require the knowledge of both the position of the PoI to cover and the characteristics of the monitored area. Obtaining all the necessary information about the environment is not an easy task, especially if the dynamic nature of the observed processes is taken into account. Furthermore, combining PoI coverage with the connectivity of each sensor with the data sink is a challenging problem in mobile sensor deployment. In this work, we address a mobile sensor deployment algorithm that combines environment and PoI discovery along with coverage and connectivity preservation. However, the PoI discovery and coverage are opposing demands if the same set of devices is used for both operations. In order to maximize the PoI coverage in the field of interest, mobile sensors have to self-deploy in a certain manner and to adjust their positions according to the placement of PoIs that need still to be discovered, which excludes the application of any standard environment exploration technique.

To the best of our knowledge, there are very few works that combine PoI discovery and coverage with connectivity preservation. Recent works focused on multi-objective mathematical models to determine the best placement of mobile nodes for different tasks [20, 27]. In [4], authors analyze mobile sensor movement on a circular path with the goal to cover the set of predefined PoIs that lay on the same path, but the position of the PoI is assumed to be known and the approach cannot be used for the multi-path problem (that introduces multiple movement paths and data sink). In [24], authors investigate the problem of collaborative area monitoring using both static and mobile sensors. They tackle the problem of unknown area exploration and coverage as well as introducing the connectivity issue. However, their focus is on a distributed algorithm for mobile sensors' path planning in order to improve the area monitoring in the way that these mobile sensors sample the areas that are least covered by the stationary sensors and move to the "suspicious" areas on stationary sensor demand in autonomous manner. A preliminary work aiming at three objectives together is [13].

In this work, we propose a novel approach that integrates the three mentioned objectives into one simple distributed sensor deployment scheme. Our approach is based on the continuous and variable speed movement of mobile sensors, which follow concentric circular paths to explore and cover the field of interest. By constantly moving, sensors execute the environment discovery task and, by adjusting the movement velocity, they satisfy the constraints on PoI coverage and connectivity with the data sink. The algorithm that runs on all the mobile sensors is distributed and introduces a new technique of velocity calculation based on the information available from the sensors in one-hop neighborhood. The contribution of this work is twofold:

- we derive analytical expressions for mobility parameters under the constraint of circular movement with the purpose of discovering and covering several PoI, while maintaining intermittent but bounded connectivity with the data sink;

- we evaluate the approach by extensive simulation campaigns in different simulation scenarios.

The rest of the paper is organized as follows: General assumptions and basic approach principle are presented in Section 2. The mathematical analysis of circular movement on concentric paths and the relationship of connectivity constraints between 
sensors and PoI coverage are presented in Section 3. The performance of our scheme is evaluated in Section 4. Section 5 overviews some of the related works. Conclusions are drawn in Section 6.

\section{General assumptions}

\subsection{Motivating application and assumptions}

Constant improvements in mobile sensor technology allows us to use different types of mobile sensors. In recent years flying drones are more and more used in military and civil applications for environmental monitoring. Interesting property of this type of mobile devices is that they consume approximately the same amount of energy while moving or staying still. Thus, it is possible to develop different dynamic deployment strategies for different types of expected coverage [37].

By using this kind of mobile sensors (which we also refer to as sensors and flying drones) that are able to move freely and without energy consumption constraints, we present a novel approach to solve the aforementioned problems by varying the velocity of the mobile device. We assume that the static sink and mobile nodes are placed or dropped in the field of interest and since the sink is static, it is considered as the center of concentric paths that will be followed by mobile sensors.

Mobile sensor velocity is calculated in a distributed manner and it is a function of the available information about PoIs, sensor characteristics and network topology. Our mobile sensor movement scenario is based on concentric circular paths with the data sink in the barycenter (Figure 1). Mobile robots move by following the predefined paths. We use circular movement for the sake of simplicity, but the approach can be used under the assumption of different closed curve configuration like ellipsoidal or hexagonal curves.

We assume that no global knowledge concerning the PoIs is available and that sensors are able to deploy themselves in order to get to their starting positions on the circular paths before the main algorithm execution. Regarding the starting positions, we analyze two cases: configuration with only one sensor per path and configuration with multiple sensors on the path.

The primary goal of our deployment scheme is the coverage of multiple PoIs in the field of interest whose positions are not known at the beginning of the deployment. By moving on the concentric paths, mobile sensors achieve PoI discovery, multiple PoI coverage and connectivity with the data sink. In the following section we define basic terminology and notation used in the remainder of the paper.

\subsection{Formal definitions}

To formally describe the deployment and coverage model of a sensor $S$, we use the Unit Disk Graph (UDG) network model [9], where sensor's communication and sensing ranges are represented with $r_{c}$ and $r_{s}$, respectively. Without loss of generality, we assume that $r_{c}>2 r_{s}$ (Figure 2(a)). The ratio between the communication and sensing range, $r_{c} / r_{s}$, is referred to as path density $\left(D_{L}\right)$. 


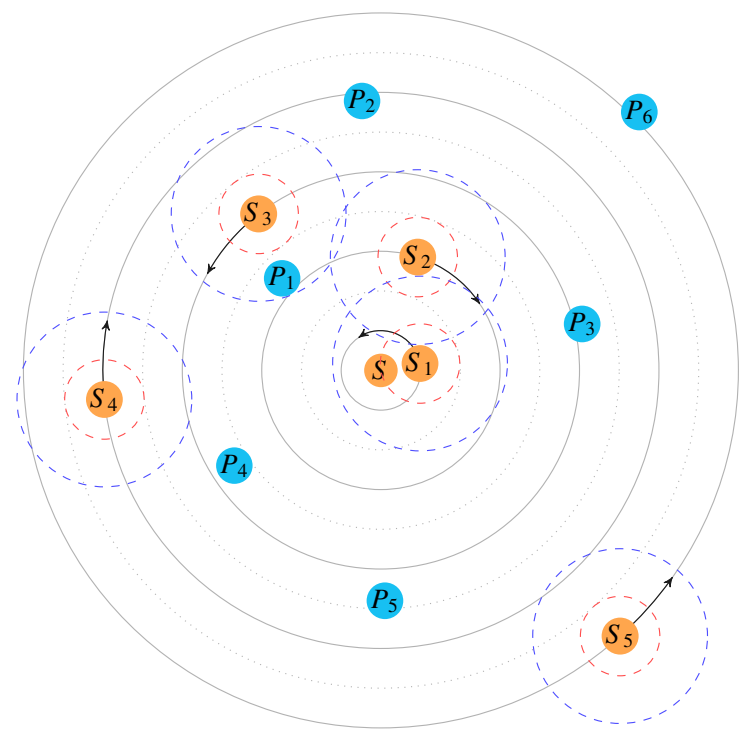

Figure 1: Mobile sensors $\left(S_{i}\right)$ follow the concentric circular paths and cover the PoIs $\left(P_{i}\right)$. Node $S$ represents the data sink.

Definition 1. The path is the closed curve that is followed by mobile sensors, denoted by $L_{n}$, where $n$ represents the number of the path or path level. Paths $L_{n}$ and $L_{n+1}$, as well as $L_{n-1}$ and $L_{n}$, are called neighboring paths.

In order to ease the calculation process, without loss of generality, we assume that all the paths are circles. The distance between neighboring paths is set to $2 r_{s}$, since this distance allows the sensors on neighboring paths to cover the area in between without overlapping (Figure 2(b)). The distance of the first path from the sink is set to $r_{s}$. Therefore, the distance of all the points of the path $L_{n}$ from the barycenter is $d_{n}=(2 n-1) r_{s}$ and the path length is $l_{n}=2 d_{n} \pi=2(2 n-1) r_{s} \pi$.

Definition 2. The stripe is the annulus referred to as $\xi_{n}, 2(n-1) r_{s}$ and $2 n r_{s}$ are its inner and outer radius, respectively. It represents the region covered by the sensor that moves following the path $L_{n}$, with the area $4(2 n-1) r_{s}^{2} \pi$.

Definition 3. The Point of Interest (PoI) is the point in the area of interest where the event occurs (denoted by $P_{i}$ in general case), defined with its polar coordinates: the distance from the data sink $r_{P}$ as the radial coordinate and the angle from the predefined direction $\Theta_{P}$ as the angular coordinate.

We assume that there is an unknown number of PoIs in the area of interest, and that the PoIs are placed in unknown locations $\left(r_{P_{i}}, \Theta_{P_{i}}\right)$. Moreover, without loss of generality, we assume that all the PoIs have equal dynamics of alternation between active and dormant states, described by the period of alternation $T_{P}$. A specific PoI is considered to be covered if it lays within the sensing range $r_{s}$ of a sensor for a period of time greater than $T_{\text {sens }}$, that is the period of time in which a sensor can get all the 


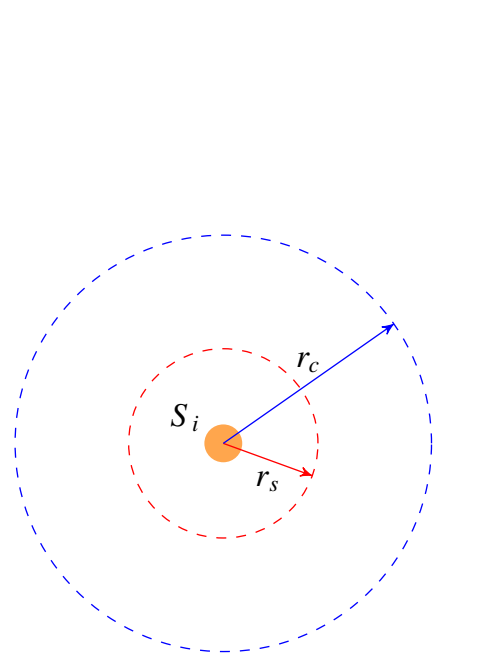

(a)

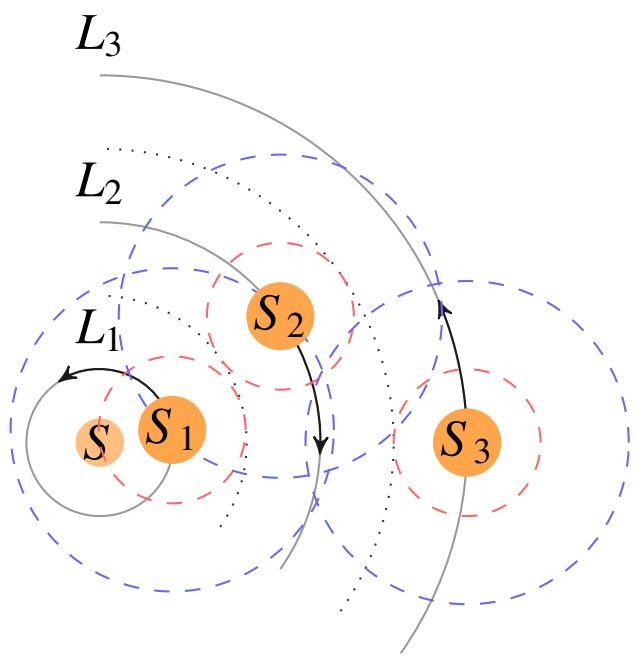

(b)

Figure 2: (a) Mobile sensor with its communication $\left(r_{c}\right)$ and sensing $\left(r_{s}\right)$ ranges. The ratio between communication and sensing range is referred to as path density, $D_{L}$. (b) The distance between neighboring paths is set to $2 r_{s}$ in order to minimize the overlap of sensing ranges.

necessary information about the event occurring at the PoI. After the information about the PoI is retrieved by the sensor, it has to be transferred to the sink in a multi-hop fashion. Similarly to $T_{\text {sens }}$, we define $T_{\text {comm }}$ as the period of time necessary to provide successful communication between two sensors. We assume $T_{\text {sens }}>T_{\text {comm }}$.

Due to the PoI dynamics, it is necessary to transfer the PoI data from a sensor to the sink as fast as possible, and therefore, we introduce the critical period of time $T_{\text {data }}$, in which the information about the PoI should be delivered to the sink from the sensor. The complete network is considered to be connected if all the messages from the sensors containing PoI information are transferred to the sink with a delay lower than $T_{\text {data }}$. In order to capture all the information regarding the PoI, a mobile sensor has to cover it with period lower than the period of PoI's dynamics $T_{P}$.

In the rest of the paper, the sensor moving on the path $L_{n}$ will be referred to as sensor $S_{n}$ or $n^{\text {th }}$ sensor. Moreover, sensors following neighboring paths are referred to as neighboring sensors.

In the following section, we analyze the dynamics of circular movements, as well as we investigate the interactions among the mobile sensors.

\section{Circular movement analysis}

In order to formally describe the movement of the sensors and the conditions of their interconnection, we first need to determine the angle between the barycenter and the positions of two sensors in the moment when they enter (or exit) each other's communication range $\left(\Theta_{\text {comm }}(n, m)\right.$, shown in Figure 3(a)). 


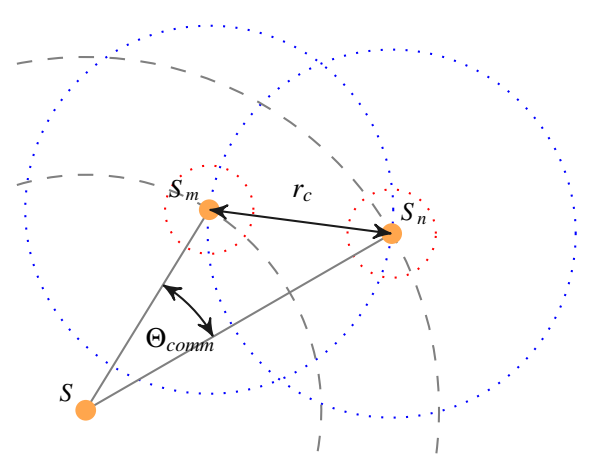

(a)

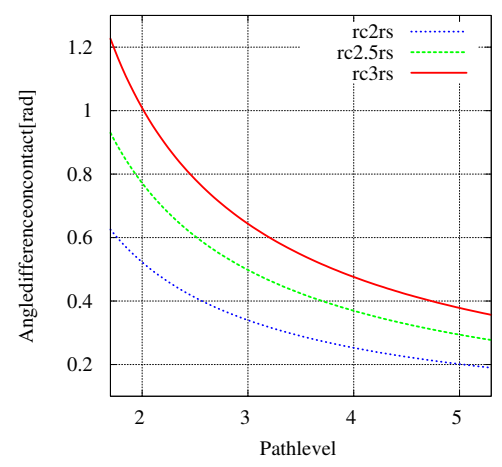

(b)

Figure 3: (a) The angle between the barycenter and two sensors on neighboring paths in the moment when they enter (or exit) each other's communication ranges. (b) Communicating sensors' angle difference between $n$ and $n-1$ path levels $\left(\Theta_{c o m m}(n, n-1)\right)$, for different values of the path density $D_{L}=r_{c} / r_{s}$.

Lemma 1. The difference between angular coordinates of sensors on paths $L_{m}$ and $L_{n}(n>m)$ in the moment of entering (or exiting) each other's communication range, denoted by $\Theta_{\text {comm }}$, is expressed as:

$$
\Theta_{c o m m}(n, m)=\arccos \frac{(2 m-1)^{2}+(2 n-1)^{2}-D_{L}^{2}}{2(2 m-1)(2 n-1)}
$$

Proof. The application of the cosines law to the triangle $S S_{n} S_{m}$ gives that $r_{c}^{2}=((2 m-$ $\left.1) r_{s}\right)^{2}+\left((2 n-1) r_{s}\right)^{2}-2(2 m-1)(2 n-1) r_{s}^{2} \cos \Theta_{c o m m}(n, m)$. Therefore, it is possible to deduce the expression for $\Theta_{\text {comm }}(n, m)$.

In the case of sensors on neighboring paths, where $m=n-1, \Theta_{\text {comm }}(n, m)$ can be simplified to $\Theta_{\text {comm }}(n)$, as presented in Equation 2. Figure 3(b) is the plot of function $\Theta_{\text {comm }}(n)$ for sensors on different paths (from path 2 to 5 , since the sensor on first path is constantly connected to the data sink) and 3 arbitrarily chosen path densities ( $D_{L} \in$ $\{2,2.5,3\})$. It is clear that an higher path density permits a larger sensor movement while preserving the connection with the communicating sensor on the neighboring path.

$$
\Theta_{\text {comm }}(n)=\frac{4 n^{2}+1-D_{L}^{2}}{4 n^{2}-1}
$$

Further in the text $\Theta_{\text {comm }}(n)$ is used for the calculation of minimal inter-contact time between mobile sensors on neighboring paths. In the following section, we analyze sensor velocity, $v_{\text {comm }}(n)$, during the communication, inter-contact period of time $T_{\text {inter }}(n)$ for both cases of movements, in the same and in opposite directions, $T_{\text {inter }}^{+}(n)$ and $T_{\text {inter }}^{-}(n)$, respectively, and PoI coverage velocity $v_{\text {sens }}(n)$.

\subsection{Movement in the same direction}

In general case, sensors move at their maximal movement velocity $v_{\max }$, and eventually slow down in the case of contact with other sensors or PoIs. Therefore, in order 
to satisfy the given communication period $T_{\text {comm }}$ and keep the connectivity, sensors in mutual contact have to travel at the communication velocity $v_{\text {comm }}(n)$. If the calculated velocity is greater than cruising velocity $v_{\max }$, then the sensor keeps $v_{\max }$ as the movement velocity, otherwise it slows down to a velocity $v_{\text {comm }}(n)<v_{\max }$.

Lemma 2. In order to satisfy the minimum communication period $T_{\text {comm }}$ during the movement on paths $L_{n}$ and $L_{n-1}$, sensors must limit their maximal velocity to:

$$
v_{\text {comm }}(n)=\frac{(2 n-1)(2 n-3) r_{s} \Theta_{c o m m}(n)}{T_{\text {comm }}}
$$

Proof. Let us consider the movement of the sensors in the same direction on paths $L_{n}$ and $L_{n-1}$. In order to satisfy the $T_{\text {comm }}$ constraint, they have to move with velocity $v_{\text {comm }}(n)$. Thus, their angular velocities are $w_{n}=v_{\text {comm }}(n) /\left((2 n-1) r_{s}\right)$ and $w_{n-1}=$ $v_{\text {comm }}(n-1) /\left((2(n-1)-1) r_{s}\right)$. The condition for keeping the connection between the sensors is $w_{\text {diff }} T_{\text {comm }}=2 \Theta_{\text {comm }}(n)$, where $w_{\text {diff }}=w_{n-1}-w_{n}$. Therefore, the maximal movement velocity that allows both sensors to keep their connection during the period $T_{\text {comm }}$ is given in Equation 3.

After the communication with sensor $S_{n-1}$ is done, sensor $S_{n}$ restores the maximal velocity $v_{\max }$ in order to minimize the inter-contact time with sensor $S_{n-1}$.

Lemma 3. Under the assumption that sensors move at velocity $v_{\max }$ after losing their connection, the needed time to establish a new connection is:

$$
T_{\text {inter }}^{+}(n)=\frac{(2 n-1)(2 n-3) r_{s}}{2 v_{\max }}\left(2 \pi-\Theta_{\text {comm }}(n)\right)
$$

Proof. Inter-contact time can be calculated by using the difference in neighboring sensors' angular coordinates, in other words, inter-contact time is the time needed to lower the sensors' angular difference to the value of $\Theta_{\text {comm }}(n)$. Neighboring sensors $n$ and $n-1$ are both moving in the same direction at the velocity $v_{\max }$, thus their angular velocities are $w_{n}=v_{\max } /\left((2 n-1) r_{s}\right)$ and $w_{n-1}=v_{\max } /\left(\left((2(n-1)-1) r_{s}\right)\right.$, respectively. The inter-contact time $T_{\text {inter }}^{+}(n)$ is the time needed for a sensor moving at the angular speed $w_{\text {diff }}=w_{n-1}-w_{n}$ to travel the angle $2 \pi-\Theta_{\text {comm }}(n)$. Since $w_{\text {diff }}=$ $2 v_{\text {max }} /\left((2 n-1)(2 n-3) r_{s}\right)$, then $T_{\text {inter }}^{+}(n)=(2 n-1)(2 n-3) r_{s}\left(2 \pi-\Theta_{\text {comm }}(n)\right) / 2 v_{\text {max }}$.

Figure 4(a) shows the inter-contact time as a function of the number of path involved, for 3 different maximal movement velocities that are chosen arbitrarily. As a consequence of the longer distance that sensors on higher paths have to travel and the nature of the movement in the same direction in the same linear velocity, inter-contact periods are unacceptably high.

\subsection{Movement in opposite directions}

To lower the inter-contact period, we use movement in opposite directions on neighboring paths. Since the connection time between two sensors on neighboring paths should be at least $T_{\text {comm }}$, in this case, their velocity should be lower or equal to $v_{\text {comm }}(n)$, where $v_{\text {comm }}(n)$ in this case is calculated similarly to Lemma 2: 


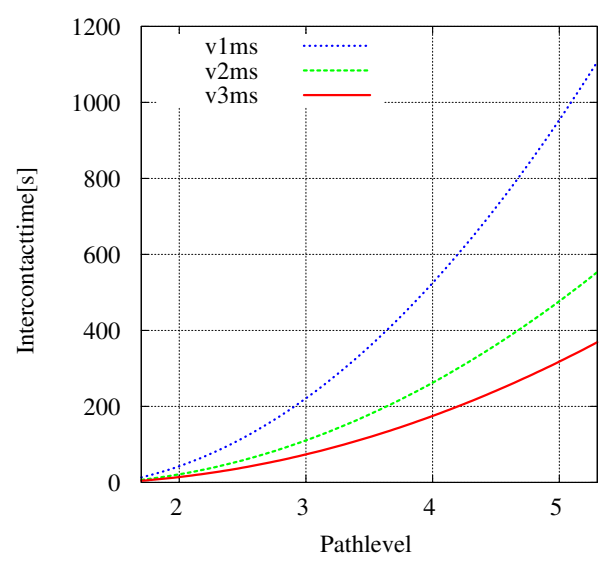

(a) Movement in the same direction.

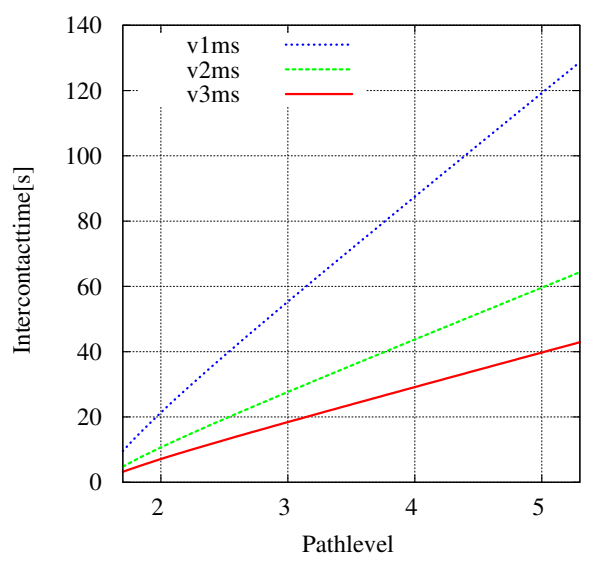

(b) Movement in opposite directions.

Figure 4: Inter-contact time for sensors on neighboring paths in the case of single sensor per path.

$$
v_{\text {comm }}(n)=\frac{(2 n-1)(2 n-3) r_{s} \Theta_{c o m m}(n)}{4(n-1) T_{c o m m}}
$$

Following the reasoning from the proof of Lemma 3, we deduce the inter-contact time for the opposite direction movement for sensors on neighboring paths (Figure 4(b)):

$$
T_{\text {inter }}^{-}(n)=\frac{(2 n-1)(2 n-3) r_{s}\left(2 \pi-\Theta_{c o m m}(n)\right)}{4(n-1) v_{\max }}
$$

\subsection{Multiple sensors on the path}

Further step in lowering the inter-contact time is the case where multiple sensors are placed on the same path (at equidistant positions). Similarly to Equations 4 and 6 , we derive the expressions for inter-contact time, both for movements in the same (Equation 7) and in the opposite directions (Equation 8), for the case where there are multiple sensors deployed on a circular path. It is worth noting that sensors on the same path always move in the same direction, differences in direction are related to the sensors on neighboring paths. The inter-contact time as a function of path number for different movement velocities is shown in Figure 5(b).

$$
\begin{aligned}
& T_{\text {inter }}^{+}(n)=\frac{(2 n-1)(2 n-3) r_{s}}{2 v_{\max }}\left(\frac{2 \pi}{n}-\Theta_{\text {comm }}\right) \\
& T_{\text {inter }}^{-}(n)=\frac{(2 n-1)(2 n-3) r_{s}}{4(n-1) v_{\max }}\left(\frac{2 \pi}{n}-\Theta_{\text {comm }}\right)
\end{aligned}
$$

In case of possible contact between the sensors following the same path, hence the sensors that move in the same direction, the sensor that senses its neighboring sensor from the same path lowers the speed thus increasing the distance between them and avoiding the collision. 


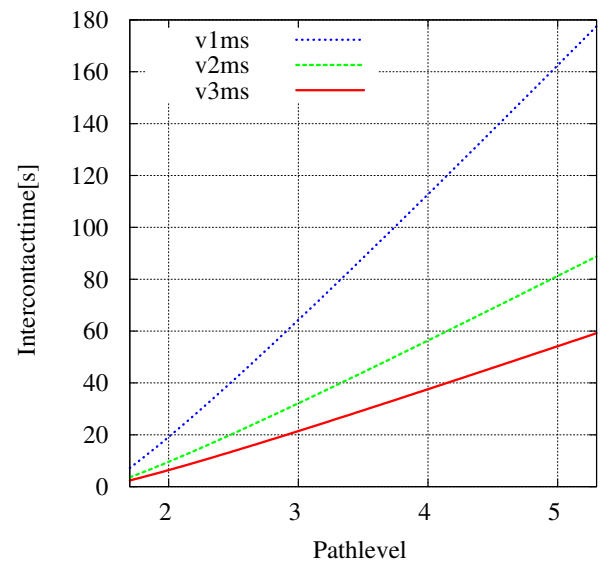

(a) Movement in the same direction.

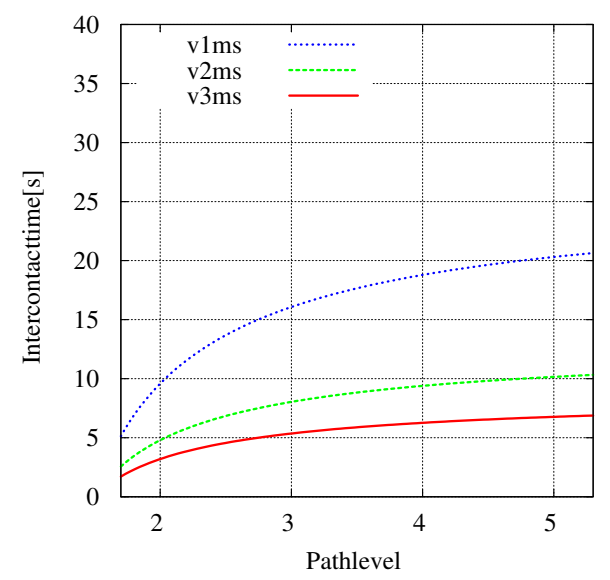

(b) Movement in opposite directions.

Figure 5: Inter-contact time for sensors on neighboring paths in the case of multiple sensors per path.

Figures 4 and 5 show significant decrease in neighboring sensor inter-contact time in the favor of movement in opposite directions with multiple sensors on a path, therefore we consider this scenario in approach evaluation (Section 4).

\subsection{Detection of a PoI}

Similarly to the case where the sensors interconnect, a sensor detecting the PoI has to change its movement velocity in order to satisfy the condition for $T_{\text {sens }}$.

Lemma 4. Depending on the PoI distance from the path $L_{n}\left(d_{P}\right)$, the angular coordinate difference $\Theta_{\text {sens }}$ (Figure 6) for the case when the PoI is on the boundary of the sensing range is:

$$
\Theta_{\text {sens }}(n)=\arccos \frac{\left(d_{n}+d_{P}\right)^{2}+d_{n}^{2}-r_{s}^{2}}{2 d_{n}\left(d_{n}+d_{P}\right)}, d_{n}=(2 n-1) r_{s}
$$

Proof. Similarly to the proof of Lemma 1, we consider the case with a sensor $S_{n}$, the base station $S$ and the PoI $P_{i}$. Applying the law of cosines to the triangle $S P_{i} S_{n}$ gives that $\left|S_{n} P_{i}\right|^{2}=\left|S S_{n}\right|^{2}+\left|S P_{i}\right|^{2}-2\left|S S_{n}\right|\left|S P_{i}\right| \cos \Theta_{\text {sens }}(n)$. Since $\left|S S_{n}\right|=(2 n-1) r_{s}$, $\left|S P_{i}\right|=(2 n-1) r_{s}+d_{P}, d_{P}=r_{P_{i}}-(2 n-1) r_{s}$ and $\left|S_{n} P_{i}\right|=r_{s}$, it is therefore $r_{s}^{2}=$ $\left((2 n-1) r_{s}\right)^{2}+\left((2 n-1) r_{s}+d_{P}\right)^{2}-2(2 n-1) r_{s}\left((2 n-1) r_{s}+d_{P}\right) \cos \Theta_{\text {sens }}(n)$.

Lemma 5. The maximal velocity of sensor movement needed to satisfy the sensing period $T_{\text {sens }}$ is:

$$
v_{\text {sens }}=\frac{2(2 n-1) r_{s} \Theta_{\text {sens }}(n)}{T_{\text {sens }}}
$$

Proof. The length of the path that sensor $S_{n}$ can travel while covering $P_{i}$ is $l=2 \Theta_{\text {sens }}(n)(2 n-$ 1) $r_{s}$. Satisfying $T_{\text {sens }}$ as the sensing constraint, the maximal movement velocity during the PoI sensing is, therefore, $v_{\text {sens }}=l / T_{\text {sens }}$. 


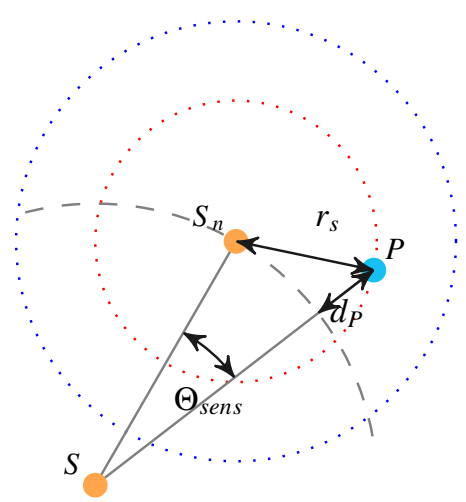

Figure 6: The difference between angular coordinates of sensor $S_{n}$ and the PoI $P_{i}$ in the moment when sensing begins.

It is worth noting that if a sensor has to choose between several values for its actual movement velocity, it chooses the smallest value, which is also the least energy consuming.

\section{Approach evaluation}

In this section we evaluate the deployment properties illustrated with the PoI discovery, network connectivity and network coverage results. The simulator used is WSNet, event driven simulator for large scale wireless networks [43]. Table 1 provides the parameters that are used in simulations. In all the simulations we test three different movements velocities: 1,2 and $3 \mathrm{~m} / \mathrm{s}$. Values for $T_{\text {comm }}$ and $T_{\text {sens }}$ are arbitrarily chosen and set to $2 s$ and $3 s$ respectively.

The positions of PoIs are chosen randomly in the given circular area, which gives the specific PoI distribution on circular paths as shown in Figure 7(a). For the simulations we take into the consideration the multiple sensor case. Specifically, we use 15 sensors placed on the paths as follows: one sensor is placed on the first, two on the second, three on the third, four on the fourth and five sensors on the fifth path. For the

Table 1: Simulation parameters

\begin{tabular}{|c|c|c|c|c|}
\hline$r_{c}[\mathrm{~m}]$ & $r_{s}[\mathrm{~m}]$ & $T_{\text {comm }}[\mathrm{s}]$ & $T_{\text {sens }}[\mathrm{s}]$ & $T_{\text {data }}[\mathrm{s}]$ \\
\hline 11 & 5 & 2 & 5 & 100 \\
\hline \hline$v_{\max }[\mathrm{m} / \mathrm{s}]$ & Area $\left[\mathrm{m}^{2}\right]$ & Duration $[\mathrm{s}]$ & Number of sensors & Number of PoIs \\
\hline $1,2,3$ & $2500 \pi$ & 600 & 15 & 50 \\
\hline
\end{tabular}

Table 2: PoI distance from the data sink and its covering sensor path level

\begin{tabular}{|c||c|c|c|c|c|}
\hline PoI distance $[\mathrm{m}]$ & $0-10$ & $10-20$ & $20-30$ & $30-40$ & $40-50$ \\
\hline Path level & 1 & 2 & 3 & 4 & 5 \\
\hline
\end{tabular}




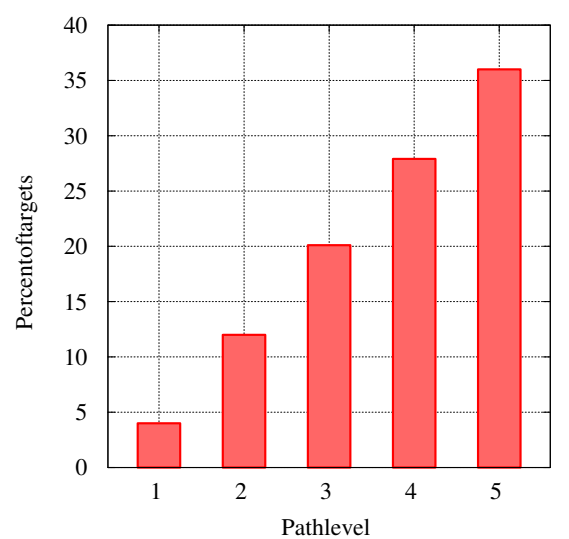

(a)

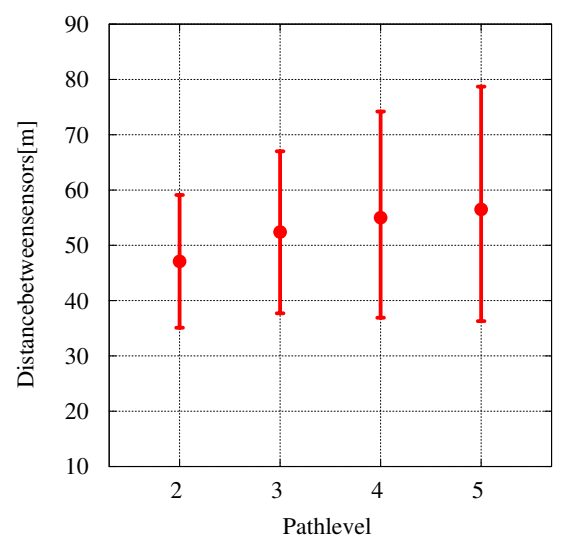

(b)

Figure 7: (a) Distribution of PoIs with relation to the path covering it and (b) the distance between the sensors on the path during the movement.

sake of clarity, Table 2 provides the reader with the relation between the PoI distance from the data sink and its covering sensor path level.

Sensors on the same path are distributed equidistantly in the beginning of the deployment, however, these distances change due to the contact with other sensors and PoIs in the field. Figure 7(b) shows the average, minimal and maximal distances between sensors on different paths.

\subsection{PoI discovery}

The PoI discovery is the first property of our proposed movement scheme. We measure the time needed to discover all the PoIs in the field of interest and compare it with the random walk approach, with the same number of sensors and their initial placement. In each simulation run, 50 PoIs are randomly deployed in the field with the area of $2500 \pi \mathrm{m}^{2}$, while the maximal sensor movement velocity varies from 1 to $3 \mathrm{~m} / \mathrm{s}$ for both random walk and our circular paths approach.

Figure 8 represents the percent of PoIs discovered after a certain time, which gives the measure of the reactivity of the network. It shows that our approach (Figure 8(a)) outperforms the random walk approach (Figure 8(b)), and that all the PoIs are discovered after $t=35 \mathrm{~s}$ and $t=56 \mathrm{~s}$ for $v_{\max }=3 \mathrm{~m} / \mathrm{s}$ and $v_{\max }=1 \mathrm{~m} / \mathrm{s}$, respectively. Random walk approach achieves the $99 \%$ coverage after $384 s$, but fails to discover all the PoIs in the field during the simulated period of time.

\subsection{Network connectivity}

After the PoI information has been captured, sensors deliver this information to the sink node by passing it to the neighboring sensor on lower path. Thus, the message delivery time can vary depending on inter-contact time between communicating sensors on neighboring paths and their initial placement on the path. Figure 9(a) shows the average message delivery time for different movement velocities when the distance 


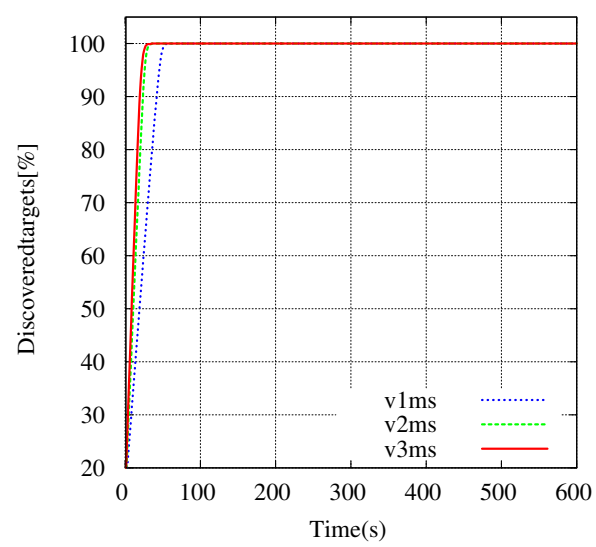

(a) Circular path approach

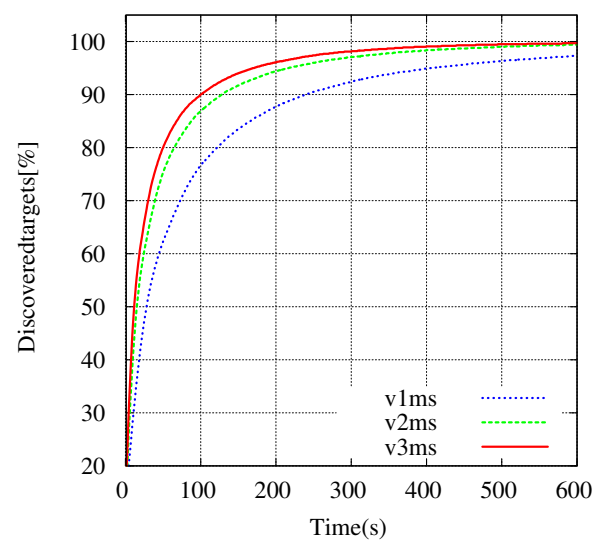

(b) Random walk approach

Figure 8: Percent of PoIs discovered after a certain time for both approaches and different movement velocities.

from the PoI and the sink varies. Step-wise delivery function that depends on distance reflects the effect of different sensors on discrete levels.

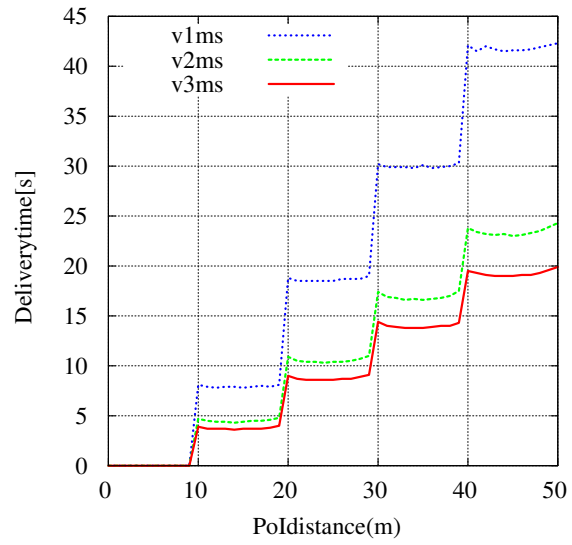

(a) PoI information delivery time

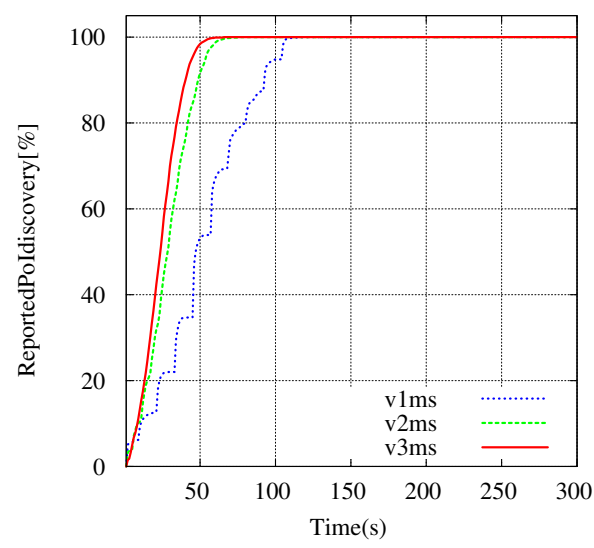

(b) Percent of reported PoIs

Figure 9: PoI information delivery time and the percent of reported PoIs to the sink node for circular path approach.

The way to observe network connectivity is to evaluate the complete PoI discovery from the sink node's point of view. In contrast to Figure 8, the PoI is considered as discovered only when the report about it arrives on the sink node. Figure 9(b) shows that the time needed to report the information about the whole field is $118 \mathrm{~s}$ for $v_{\max }=$ $1 \mathrm{~m} / \mathrm{s}$ and $66 \mathrm{~s}$ for $v_{\max }=3 \mathrm{~m} / \mathrm{s}$.

Another way to evaluate the connectivity of the sensors with the sink, and thus the 


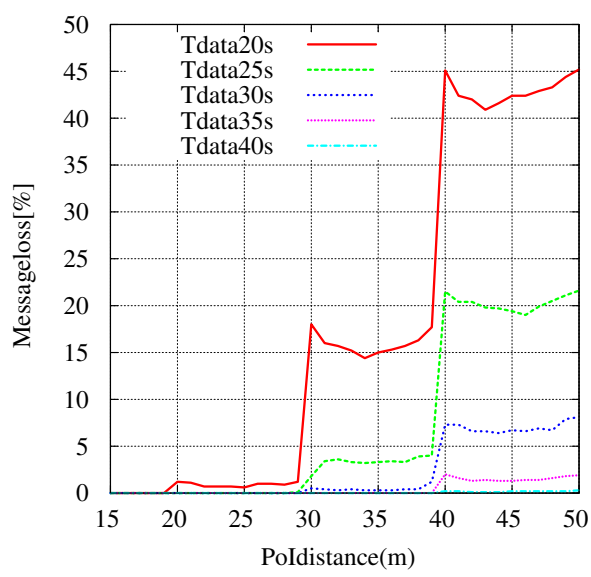

(a) Message loss with relation to the PoI distance from the sink

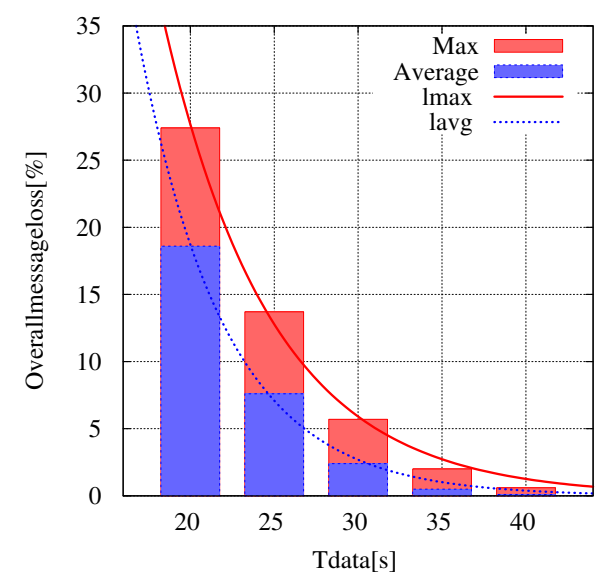

(b) Overall message loss with relation to $T_{\text {data }}$

Figure 10: Message loss related to $T_{\text {data }}$ in circular path approach.

connectivity of the complete network, is to verify the fraction of lost messages due to the expiration of the message lifetime timer $T_{\text {data }}$. In this set of simulations, we observe the message loss with relation to the PoI distance from the sink (Figure 10(a)) and the overall message loss during the simulation time (Figure 10(b)) for different values of $T_{\text {data }} \in\{20,25,30,35,40\}$. Figure 10(a) shows that fourth and fifth level sensors are critical for all the values of $T_{\text {data }}$, where the message loss reaches $17 \%$ for $L_{4}$ and $45 \%$ for $L_{5}$. The maximal movement velocity is assumed to be $v_{\max }=3 \mathrm{~m} / \mathrm{s}$.

The relation between the overall message loss and $T_{\text {data }}$ is presented in Figure 10(b), from which we can deduce the minimal value of $T_{\text {data }}$ needed to obtain the message delivery to the sink node with certain message loss threshold. Furthermore, we provide the expressions for overall average $l_{\text {avg }}$ and maximal message loss $l_{\text {max }}$, that can be used for message loss prediction for a given $T_{\text {data }}$ (Equation 11).

$$
l_{\text {avg }}=906.679 e^{-\left(T_{\text {data }} / 5.1541\right)} \quad l_{\max }=608.074 e^{-\left(T_{\text {data }} / 6.4732\right)}
$$

\subsection{PoI coverage}

The PoI coverage is evaluated by analyzing the PoI update periods and the overall coverage time (depending on the PoI distance from the sink). The update period is the time interval between two consecutive detections of the same PoI. Figure 11 shows the comparison of PoI update period with relation to PoI distance from the sink, for both random walk and circular scenario. It is worth noting that our approach achieves short update periods. Thus, the frequency of PoI visits and reactivity of the whole network increase. It is also worth noting here that PoIs included into consideration are only discovered PoIs (that means all the PoIs for our approach, and only a fraction of all the PoIs for the random walk approach).

Local maximums in the Figure 11(a) are the update periods for the PoIs located on the edge of sensors' sensing range. Lower PoI update periods, in the same figure, come 


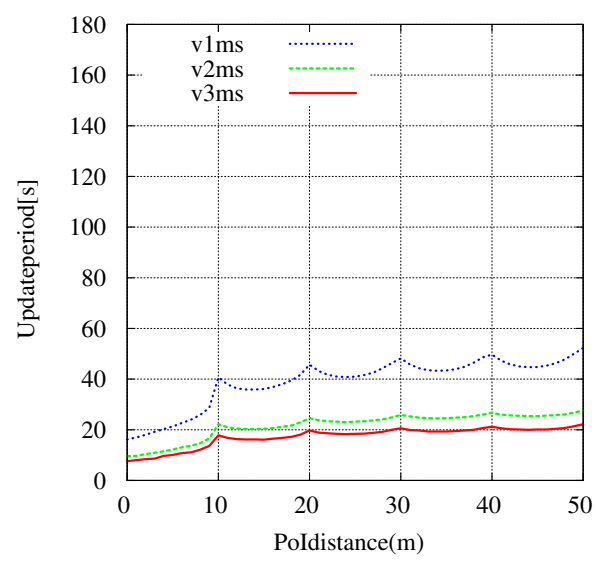

(a) Circular path approach

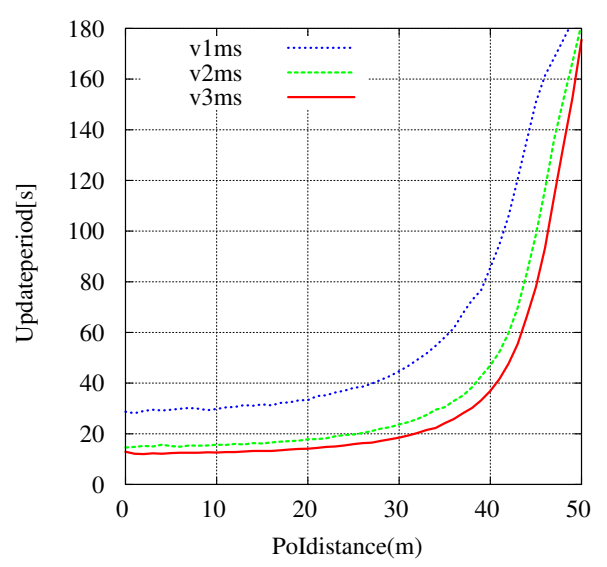

(b) Random walk approach

Figure 11: PoI update time with relation to different movement velocities.

from PoIs located next to the center of the path described by the sensors. The update time for the random walk approach (Figure 11(b)) increases with the PoI distance from the sink node. Low update periods for PoIs located closed to the sink are the result of the sensors that move randomly and thus statistically more often traverse the central area of the region of interest.

Figure 12 presents the PoI coverage evaluation, the percent of time spent while covering the PoI with relation to the PoI distance from the sink for three sensor movement velocities, $v_{\max } \in\{1,2,3\}$. Due to the $T_{\text {sens }}$ condition for covering the PoIs on the edge of the path, other PoIs are covered longer since the covering sensors covers them at the same time as the PoI on the edge. This case results in local maximums shown in Figure 12(a). Coverage time for all the PoIs is the highest for the highest movement speed, $v_{\max }=3 \mathrm{~m} / \mathrm{s}$. Random walk approach coverage decreases with the PoI distance from the sink, as opposing to the update period graphs. It is worth noting that the fraction of discovered PoIs during the simulation time in the random walk approach is lower than that in the circular path approach.

The percent of time covered also depends on the number of PoIs in the field of interest. This relation is shown in Figure 13(a) for circular paths and Figure 13(b) for random walk approach. Figures show that the percent of time spent in PoI coverage decreases as the number of PoIs increases due to the number of PoIs that need to be processed during the movement. Higher PoI density requires slower sensor movement and thus produces the output similar to the movement with lower velocity (shown in Figure 12).

\section{Related works}

Our work covers three different operational phases of Mobile Wireless Sensor Networks, specifically exploration of the sensor field and discovery of the Points of Interest 


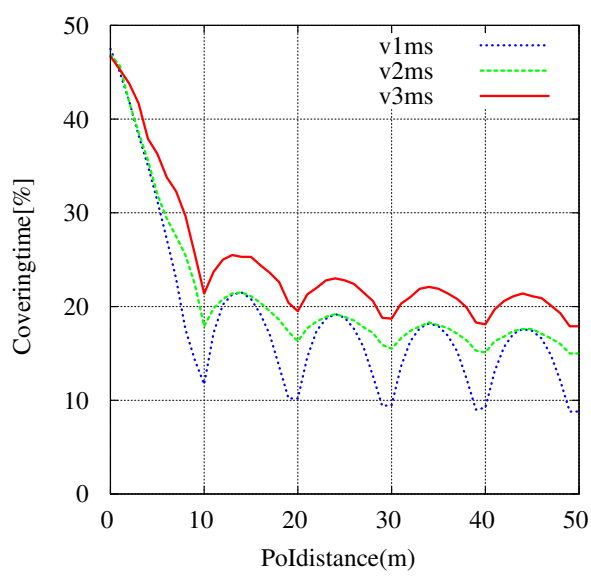

(a) Circular path approach

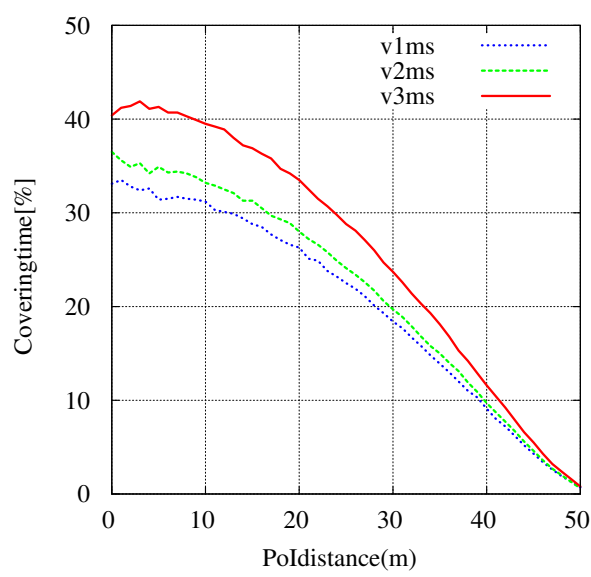

(b) Random walk approach

Figure 12: PoI coverage with relation to different movement velocities.

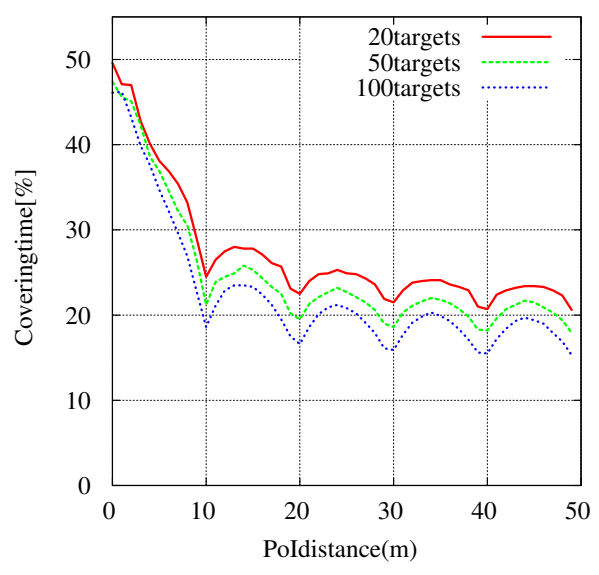

(a) Circular path approach

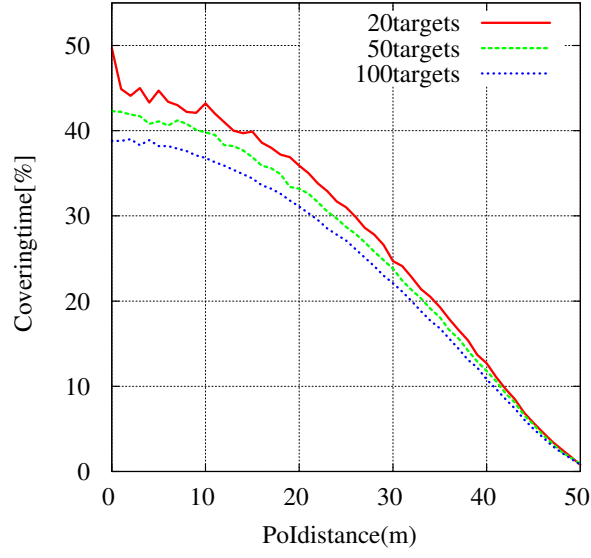

(b) Random walk approach

Figure 13: Covering time with relation to different number of PoIs.

(PoI), monitoring of and data gathering from the PoIs, connection with and data reporting to the Base Station. In this section we analyze only the most recent works referred to these three main issues.

\subsection{Discovery}

The exploration of a geographical area by a group of autonomous agents looking for Points of Interest has been widely studied in the literature of robotics. In fact, many variations of this task have already been considered. For sake of synthesis we will mention only few works from the extensive literature in this area, in order to highlight 
the placement of the discovery phase of our approach. The basic difference of our approach with respect to the existing works is that, in our case, mobile devices exploit their communications capabilities to coordinate in order to fully explore the area and proceed covering the assigned area with no need of further formation control. In literature we can find works aiming at the same purpose by using a single mobile robot, as in [30], where the authors propose an energy-efficient technique to determine the trajectories to choose in order to minimize the total travelled distance. Of course, also more sophisticated works have been presented for the PoI discovery task. In these works the number of mobile devices is higher and coordination issues arise. Usually, in the robotics community, communication capabilities are seen as an unnecessary and unwanted supplement of the basic devices' facilities, as in [18] and [28], where robots do not have any communication facility to exploit, and in [21] where also the perception capabilities of the devices is restricted. Few works consider the possibility for the nodes to communicate and exchange useful information, as in [15], but even in these cases the communication is limited and relayed by a central unit. In our work, the mobile devices use their communication capabilities during all the phases of the algorithm, both to coordinate during the initial formation control and to relay the information about the monitored events towards the base station. Furthermore, robotics works on formation control, please refer to [42] for a survey, assume continuous communications among the devices are necessary in order to support the formation control, on the contrary, in our work we assume that devices communicate only at the beginning of the discovery phase, in order to coordinate, to avoid overlaps of their coverage areas and to distribute on the whole field.

\subsection{Connectivity}

We refer to the third phase of our scheme with the term connectivity, because we investigate both theoretically and by simulations the impact of devices mobility on connection times. More precisely, we are interested in evaluating the performance of both the upper layer parameters, through the measurement of the communication delay between each node and the base station, and the one-hop communication, such as the inter-contact time among neighboring nodes. A lot of efforts have been already produced on Delay Tolerant Networks (DTN) when mobility of nodes is taken into account. In [40], authors show that mobility increases connectivity in k-hop clustered networks, and significantly improves the network lifetime and the power-delay tradeoff. As usual when authors aim at demonstrating some interesting properties achieved by letting nodes move, the mobility follows one of the classical schemes, specifically the random walk mobility model with non trivial velocity. Instead, in our work, we assume that nodes can control their mobility according to surrounding conditions. Even in [16], authors show the positive impact of mobility on the determination of delaythroughput trade off in MANET. Even in this case, nodes' mobility is restricted to movement around nodes' own home-points. In [32], the mobility is controlled and nodes move from the initial configuration towards a spatial distribution that increases the network's throughput capacity and decreases the mean-service delay of packets. A similar approach is used in [10] to determine the optimal placement in terms of energy consumption by virtualizing nodes' movements. In these works, upon the determination of the optimal placement, nodes do not have to move further. In our case, the 
coverage of the area requires nodes to keep moving for the whole network lifetime. Please refer to [17] for the survey on sensing coverage and network connectivity in WSN.

\subsection{Coverage}

The coverage and monitoring of a PoI, an area of interest or the whole sensor field is a subject covered from both the ad hoc and sensor and the robotics community by using different approaches and by focussing on different aspects. While the ad hoc and sensor community considers devices such as sensors and actors, whose task computational and execution capabilities are limited, but have the possibility to communicate with each other in wireless, the robotics community takes into account smarter devices and assumes that communications do not have a basic importance in achieving the coverage of the area. Since the topic has been extensively treated by both the communities in recent years, we will focus on the efforts produced by the ad hoc and sensor community, which are more relevant for our work. In [8], [37] and [44], authors survey coverage path planning algorithms for mobile robots, movement strategies for improving network coverage and general strategies and techniques for node placement, respectively. They propose to classify works according to the targeted coverage. Specifically, full coverage aims at cover completely the field by geographically distributing sensors and actors on the entire field according to same technique in order to continuously monitor the field. In this group of works, [29] combines computational geometry and graph theoretic techniques to determine the optimal polynomial time worst and average case algorithm for coverage calculation; in [22] the number of sensor to deploy is achieved in order to guarantee k-coverage of the fields at all times and maximize the lifetime; in [26], authors propose an algorithm of topology control, by varying the radius of the sensing range in order to maintain the coverage of the whole field when the network topology varies. The last cited work considers a limited mobility of the nodes, in [38] and [41], mobile nodes are used to extend the capability of a static sensor network. Authors of [39] and [45] use mobility of the nodes in a more extensive way, in fact they propose sensor deployment and target localization, respectively, by using virtual forces. Our approach also considers a large use of mobile nodes as the last two cited works, but the main difference is in the algorithm that drives nodes movements, we do not use virtual forces in order not to limit the mobility of nodes only to the attraction-repulsion mechanisms. The second group of works regards the barrier coverage, which is the formation of a barrier through sensors movements to monitor a specific area, usually a strip. Since our work differs substantially from the barrier coverage, we mention only [23], which proposed the first theoretical study of this problem and [6] that contains an interesting localized algorithm. In our work, we are more interested in PoI coverage and area coverage, which has been treated in literature through the usage of static nodes, as in [5] and [36] that organize the network by using clusterization and set covers to improve its lifetime. When a PoI (or an area of interest) does not need to be covered at all the time and is time-variant, the problem can be classified as sweep coverage. Robotics community also considered the sweep coverage, for example in [2]. Basically, sweep coverage allows nodes to move on the field, because the requested coverage of a PoI is limited in time. In [25], authors propose a theoretical study to prove that the determination of the minimum number of required sensors is 
NP-Hard and propose three variants of an algorithm that solves the sweep coverage problem. In respect of the last cited work that focuses only on the coverage part, we propose an algorithm that aims not only at covering the time-variant PoIs but takes into consideration also the discovery phase and the data report to the base station.

\subsection{Discovery, connectivity and coverage combined}

Finally, we analyze few works that present a combination of the mentioned objectives. The swarm intelligence community is very active in coordination of mobile agents by mimicking natural systems. This methodology is useful for making nodes perform the exploration of the field while they maintain connectivity with each other [12]. Usually for these purposes, hybrid network architectures are considered, as in the cited paper that uses two kinds of mobile robots: wheeled and flying. In our work, we assume to use homogeneous devices which are not specialized in a specific task. In a less recent work, the problem of achieving full coverage of a field while preserving 2connectivity ${ }^{1}$ among the nodes is considered [1]. As it is clear, the final objective is the deployment of the nodes, therefore further movements of the nodes after the optimal placement is achieved, are not considered. At last, we report a work from the robotics community that considers PoI discovery and coverage of the network [3]. The discovery phase is performed by mobile robots and driven by a network of radio beacons which assists the robot(s) also during the coverage. On the contrary, our scheme does not consider any additional pre-deployed hardware for achieving the same objectives.

\section{Conclusion}

In this work we proposed a novel approach to integrate PoI discovery, multiple PoI coverage and data report to the sink. Our motivation for this work is the application of flying mobile sensors for the environmental monitoring, where there is a need to gather as much information as possible while covering the events that occur in the field of interest. By constantly moving, sensors execute the environment discovery task, and by adjusting the movement velocity, they satisfy the constraints regarding the PoI coverage and $T_{\text {data }}$ connectivity with the data sink in order to report the PoI data. We have analyzed the effectiveness of the proposed approach analytically and have provided extensive simulation results to prove the feasibility of our concept.

Future work on this topic will be based on different mobile sensor deployment algorithms and topologies that can be deduced from the proposed one. Furthermore, we will include more realistic network models along with the implementation on real mobile sensors.

\section{Acknowledgements}

This work was partially funded by the French National Research Agency under the grant number: ANR VERSO RESCUE (ANR-10-VERS-003).

\footnotetext{
${ }^{1}$ a $k$-connected network is a network where each node is at least connected to $k$ neighbors.
} 


\section{References}

[1] X. Bai, S. Kumar, D. Xuan, Z. Yun, T. H. Lai, Deploying wireless sensors to achieve both coverage and connectivity, Proceedings of the 7th ACM international symposium on Mobile ad hoc networking and computing (MobiHoc '06), 2006, pp. 131-142.

[2] M. A. Batalin, G. S. Sukhatme, Multi-robot dynamic coverage of a planar bounded environment, Proceedings of the International Conference on Intelligent Robots and Systems (RSJ), 2002.

[3] M. A. Batalin, G. S. Sukhatme, Coverage, exploration and deployment by a mobile robot and communication network, Proceedings of the International Workshop on Information Processing in Sensor Networks (IPSN '03), 2003, pp. 376391.

[4] N. Bisnik, A. Abouzeid, V. Isler, Stochastic event capture using mobile sensors subject to a quality metric, Proceedings of the 12th ACM annual international conference on Mobile computing and networking (MobiCom '06), 2006, pp. 98109.

[5] M. Cardei, D. Du, Improving wireless sensor network lifetime through power aware organization, ACM Wireless Networks 29, 2005, pp. 333-340.

[6] A. Chen, S. Kumar, T. H. Lai, Designing localized algorithms for barrier coverage, Proceedings of the 13th annual ACM international conference on Mobile computing and networking (MobiCom '07), 2007, pp. 63-74.

[7] X. Chen, K. Makki, K. Yen, N. Pissinou, Sensor network security: a survey, IEEE Communications Surveys Tutorials 11 (2), 2009, pp. 52-73.

[8] H. Choset, Coverage for robotics - A survey of recent results, Annals of Mathematics and Artificial Intelligence 31 (1-4), 2001, pp. 113-126.

[9] B. N. Clark, C. J. Colbourn, D. S. Johnson, Unit disk graphs, Discrete Mathematics 86 (1-3), 1990, pp. 165-177.

[10] C. Costanzo, V. Loscrí, E. Natalizio, Distributed virtual-movement scheme for improving energy efficiency in wireless sensor networks, Proceedings of the 12th ACM international conference on Modeling, analysis and simulation of wireless and mobile systems (MSWiM '09), 2009, pp. 297-304.

[11] C. Costanzo, V. Loscrí, E. Natalizio, T. Razafindralambo, Nodes self-deployment for coverage maximization in mobile robot networks using an evolving neural network, Computer Communications 35 (9), 2012, pp. 1047-1055.

[12] F. Ducatelle, G. A. Di Caro, L. M. Gambardella, Cooperative self-organization in a heterogeneous swarm robotic system, Proceedings of the 12th annual ACM conference on Genetic and evolutionary computation (GECCO '10), 2010, pp. $87-94$. 
[13] M. Erdelj, E. Natalizio, T. Razafindralambo, Multiple point of interest discovery and coverage with mobile wireless sensors, Workshop on Mobility and Communication for Cooperation and Coordination (MC3) at International Conference on Computing, Networking and Communications (ICNC '12), 2012.

[14] M. Erdelj, T. Razafindralambo, D. Simplot-Ryl, Covering points of interest with mobile sensors, IEEE Transactions on Parallel and Distributed Systems 24 (1), 2013, pp. 32-43.

[15] D. Fox, J. Ko, K. Konolige, B. Limketkai, D. Schulz, B. Stewart, Distributed multirobot exploration and mapping, Proceedings of the IEEE 94 (7), 2006, pp. $1325-1339$.

[16] M. Garetto, E. Leonardi, Restricted mobility improves delay-throughput tradeoffs in mobile ad-hoc networks, IEEE Transactions on Information Theory 56 (10), 2010, pp. 5016-5029.

[17] A. Ghosh, S. K. Das, Coverage and connectivity issues in wireless sensor networks: A survey, Pervasive and Mobile Computing 4 (3), 2008, pp. 303-334.

[18] C. Gifford, R. Webb, J. Bley, D. Leung, M. Calnon, J. Makarewicz, B. Banz, A. Agah, Low-cost multi-robot exploration and mapping, Proceedings of the IEEE international conference on Technologies for Practical Robot Applications (TePRA '08), 2008, pp. $74-79$.

[19] N. Gouvy, E. H. Elhafsi, N. Mitton, D. Simplot-Ryl, Minimising energy consumption through mobility with connectivity preservation in sensor networks, IJPEDS 27 (6), 2012, pp. 521-540.

[20] F. Guerriero, A. Violi, E. Natalizio, V. Loscrí, C. Costanzo, Modelling and Solving Optimal Placement problems in Wireless Sensor Networks, Elsevier Applied Mathematical Modelling 35 (1), 2011, pp. 230-241.

[21] S. Guilbalt, A. Pelc, Gathering asynchronous oblivious agents with local vision in regular bipartite graphs, Proceedings of the 18th international colloquium on Structural Information and Communication Complexity (SIROCCO 2011), 2011, pp. 162-173.

[22] S. Kumar, T. H. L., A. Arora, On k-coverage in a mostly sleeping sensor network, Proceedings of the 11th annual international conference on Mobile computing and networking (MobiCom '04), 2004, pp. 144-158.

[23] S. Kumar, T. H. Lai, A. Arora, Barrier coverage with wireless sensors, Proceedings of the 11th annual ACM international conference on Mobile Computing and networking (MobiCom '05), 2005, pp. 284-298.

[24] T. P. Lambrou, C. G. Panayiotou, Collaborative area monitoring using wireless sensor networks with stationary and mobile nodes, EURASIP Journal on Advances in Signal Processing 2009 (7), 2009, pp. 1-16. 
[25] M. Li, W. C. K. Liu, Y. He, X.-Y. Li, X. Liao, Sweep coverage with mobile sensors, IEEE Transactions on Mobile Computing 10, 2011, pp. 1534-1545.

[26] L. Lin, H. Lee, Distributed algorithms for dynamic coverage in sensor networks, Proceedings of the 26th ACM annual symposium on Principles of Distributed Computing (PODC '07), 2007, pp. 392-393.

[27] V. Loscrí, E. Natalizio, C. Costanzo, F. Guerriero, A. Violi, Optimization Models for Determining Performance Benchmarks in Wireless Sensor Networks, Proceedings of IARIA SENSORCOMM, 2009.

[28] D. Massaguer, C. L. Fok, N. Venkatasubramanian, G. C. Roman, C. Lu, Exploring sensor networks using mobile agents, Proceedings of the 5th international joint conference on Autonomous agents and multiagent systems (AAMAS '06), 2006, pp. 323-325.

[29] S. Meguerdichian, F. Koushanfar, M. Potkonjak, M. Srivastava, Coverage problems in wireless ad-hoc sensor networks, Proceedings of the 20th IEEE Annual Joint Conference of the IEEE Computer and Communications Societies (INFOCOM '01), 2001, pp. 1380-1387.

[30] Y. Mei, Y.-H. Lu, C. Lee, Y. Hu, Energy-efficient mobile robot exploration, Proceedings of the IEEE International Conference on Robotics and Automation (ICRA '06), 2006, pp. $505-511$.

[31] N. Mitton, B. Sericola, S. Tixeuil, E. Fleury, I. G. Lassous, Self-stabilization in self-organized wireless multihop networks?, Ad Hoc \& Sensor Wireless Networks 11 (1-2), 2011, pp. 1-34.

[32] T. Nadeem, S. Parthasarathy, Mobility control for throughput maximization in ad-hoc networks: Research articles, Wireless Communications and Mobile Computing 6, 2006, pp. 951-967.

[33] E. Natalizio, V. Loscrí, Controlled Mobility in Mobile Sensor Networks: Advantages, Issues and Challenges, Springer Telecommunication Systems, 2011, DOI: 10.1007/s11235-011-9561-x.

[34] Z. Shi, C. Beard, K. Mitchell, Analytical models for understanding space, backoff, and flow correlation in CSMA wireless networks, Wireless Networks, 2012, pp. 1-17.

[35] Z. Shi, C. C. Beard, K. Mitchell, Competition, cooperation, and optimization in multi-hop CSMA networks with correlated traffic, IJNGC 3 (3), 2012.

[36] S. S., M. Potkonjak, Power efficient organization of wireless sensor networks, Proceedings of the IEEE International Conference on Communications (ICC '01), 2001, pp. 472-476.

[37] B. Wang, H. B. Lim, D. Ma, A survey of movement strategies for improving network coverage in wireless sensor networks, Computer Communications 32 (1314), 2009, pp. 1427-1436. 
[38] D. Wang, J. Liu, Q. Zhang, Probabilistic field coverage using a hybrid network of static and mobile sensors, Proceedings of the 15th IEEE International Workshop on Quality of Service, 2007, pp. 56-64.

[39] G. Wang, G. Cao, T. F. L. Porta, Movement-assisted sensor deployment, IEEE Transactions on Mobile Computing 6 (5), 2006, pp. 640-652.

[40] Q. Wang, X. Wang, X. Lin, Mobility increases the connectivity of k-hop clustered wireless networks, Proceedings of the 15th ACM annual international conference on Mobile Computing and networking (MobiCom '09), 2009, pp. 121-132.

[41] W. Wang, V. Srinivasan, K.-C. Chua, Coverage in hybrid mobile sensor networks, IEEE Transactions on Mobile Computing 7, 2008, pp. 1374-1387.

[42] Z. Wang, Formation control in mobile actuator/sensor networks, Spie, 2005, pp. 706-717.

[43] WSNet - An event-driven simulator for large scale wireless sensor networks, http://wsnet.gforge.inria.fr.

[44] M. Younis, K. Akkaya, Strategies and techniques for node placement in wireless sensor networks: A survey, Ad Hoc Networks 6 (4), 2008, pp. 621-655.

[45] Y. Zou, K. Chakrabarty, Sensor deployment and target localization based on virtual forces, Proceedings of the 22nd Annual Joint Conference of the IEEE Computer and Communications (INFOCOM '03), 2003, pp. 1293-1303. 